\title{
CONTINUATION OF RIEMANN SURFACES
}

\section{RICH ARD ROCHBERG ${ }^{1}$}

ABSTRACT. It is known that a nonplanar Riemann surface cannot be continued into all compact Riemann surfaces of a fixed positive genus. The Poincaré metric is used to construct a conformal invariant which is used to give an essentially geometric proof of this result.

1. Introduction. If there is a univalent analytic map of the Riemann surface $R$ into the Riemann surface $S$, we say that $R$ can be continued into S. A necessary condition to insure that a Riemann surface can be continued into all compact Riemann surfaces of a given positive genus was given by Heins [2].

Theorem 1. If the Riemann surface $R$ can be continued into all compact Riemann surfaces of given positive genus, then $R$ is conformally equivalent to a bounded plane region.

The proof consists of showing first that an open planar subregion of a compact Riemann surface of positive genus is conformally equivalent to a bounded plane region; second, that if for some $g, g>1, R$ can be continued into all compact surfaces of genus $g$ then $R$ can be continued into all compact surfaces of genus 1; and finally that any surface which can be continued into all compact surfaces of genus 1 must be planar. The proof yields a bit more than Theorem 1. It shows that if $R$ can be continued into certain surfaces of genus $g$ which cover surfaces of genus 1 of arbitrarily large module then $R$ is planar.

We will exhibit a specific geometric limitation on the class of Riemann surfaces into which a given nonplanar Riemann surface can be extended. This will allow a strengthening of Theorem 1 and will yield a proof which emphasizes the essentially geometric nature of the result.

Presented to the Society, January 15, 1974; received by the editors May 18, 1973 and, in revised form, January 10, 1974.

AMS (MOS) subject classifications (1970). Primary 30-00, 30A30, 30A46.

1 This research was supported in part by National Science Foundation Grant GP-34628. 
2. The size of a Riemann surface. For any Riemann surface $S$ which has the unit disk as its universal covering surface and $\gamma$ a curve on $S$, denote by $|\gamma|_{S}$ the length of $\gamma$ with respect to the Poincare metric on $S$. Let $S$ be such a surface. For $A$ an annulus in $S$, a subset of $S$ homeomorphic to a closed annulus, define $|A|_{S}=\inf \left\{|\gamma|_{S} ; \gamma\right.$ a curve in $A$ which connects the two components of the boundary of $A\}$. Say that a set of annuli $\left\{A_{i}\right\}$ reduces $S$ if the $A_{i}$ are disjoint annuli in $S$ and $S \backslash \bigcup_{i} A_{i}$ is planar. Note that the number of annuli needed to reduce $S$ equals the genus of $S$. For any such $S$ we define the size of $S$ by

$$
\operatorname{size}(S)=\sup \left\{\inf _{i}\left|A_{i}\right| s ;\left\{A_{i}\right\} \text { reduces } S\right\} \text {. }
$$

If $S$ is nonplanar and does not have the unit disk as its universal covering surface, then $S$ is a compact surface of genus 1 . Such $S$ has a transitive group of conformal automorphisms [4, Volume I, Chapter I]. Hence, for such an $S$ and $p$ in $S$, size $(S \backslash\{p\})$ is independent of the choice of $p$. In this case we define size $(S)$ to be size $(S \backslash\{p\})$.

The function size $(S)$, now defined for all nonplanar $S$, can be used to describe a geometric constraint on continuation of Riemann surfaces.

Proposition 1. A nonplanar Riemann surface cannot be continued into Riemann surfaces of arbitrarily large size.

Proof. Let $R$ be a nonplanar Riemann surface. Since a compact surface has no nontrivial continuations, we may assume $R$ is noncompact. In particular, $R$ carries a Poincaré metric. Since $R$ is nonplanar we can choose $\gamma_{1}$ and $\gamma_{2}$ to be two smooth simple closed curves on $R$ which cross exactly once. Let $S$ be any nonplanar surface into which $R$ can be continued and $f$ a univalent analytic map of $R$ into $S$. If $S$ is a compact surface of genus 1 then $f$ also maps $R$ into a surface $S^{\prime}$ of the same size as $S$ obtained by removing from $S$ any point not in $f(R)$. Hence we may assume $S$ carries a Poincaré metric. Let $\left\{A_{i}\right\}$ be any set of annuli which reduces $S$. The curve $f\left(\gamma_{1}\right) \cup f\left(\gamma_{2}\right)$ must contain a collection of arcs which form a curve in some $A_{i}$ connecting the two boundary components of that $A_{i}$. To see this, note that otherwise the curve $\gamma_{1} \cup \gamma_{2}$ would be mapped smoothly and univalently into the planar domain formed by adjoining to the planar domain $S \backslash\left(\bigcup_{i} A_{i}\right)$ the components of each $A_{i}$ cut off by $f\left(\gamma_{1}\right) \cup f\left(\gamma_{2}\right)$. However, a planar domain cannot carry a pair of simple closed curves which crosses exactly once. Hence $\left|A_{i}\right|_{S} \leq\left|f\left(\gamma_{1}\right) \cup f\left(\gamma_{2}\right)\right|_{S^{*}}$. By the Schwarz-Pick lemma, 
$\left|f\left(\gamma_{1}\right) \cup f\left(\gamma_{2}\right)\right|_{S} \leq\left|\gamma_{1}\right|_{R}+\left|\gamma_{2}\right|_{R}$. Thus inf $\left.\left.{ }_{j}|| A_{j}\right|_{S}\right\} \leq\left|\gamma_{1}\right|_{R}+\left|\gamma_{2}\right|_{R}$. Since the choice of reducing set was arbitrary, size $(S) \leq\left|\gamma_{1}\right|_{R}+\left|\gamma_{2}\right|_{R}$. The proposition is proved.

Corollary. A Riemann surface which can be continued into surfaces of arbitrarily large size is planar.

3. Surfaces of large size. To apply the previous result it is necessary to know that surfaces of large size exist.

Proposition 2. Let $K$ be a positive number and $g$ a positive integer. There is a compact Riemann surface $S$ of genus $g$ with size $(S)>K$.

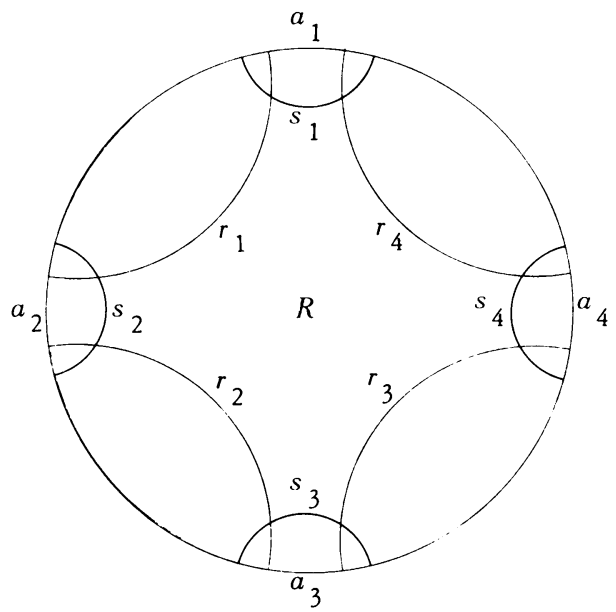

Figure 1

Proof. First we construct such a surface for $g \geq 2$ (for $g=2$ the construction is summarized in Figure 1). Let $D$ be the open unit disk of the complex plane endowed with the noneuclidean geometry given by the Poincare metric. For $j=1, \cdots, 2 g$ let $a_{j}=\exp (\pi j i / g)$. Pick $\alpha, 0<\alpha<\pi / 4 g$. For $j=1, \cdots, 2 g$, let $r_{j}$ be the noneuclidean straight line in $D$ joining $a_{j} \exp (i \alpha)$ to $a_{j}(\exp i(\pi / g-a))$. For $\beta$ with $a<\beta<\pi / 4 g$, for $j=1, \cdots, 2 g$, let $s_{j}$ be the noneuclidean straight line in $D$ joining $a_{j} \exp (i \beta)$ to $a_{j} \exp (-i \beta)$. These $4 g$ noneuclidean straight lines divide the disk into a finite number of (not necessarily bounded) noneuclidean polygons. The central polygon is a fundamental domain for the surface we wish to construct. Let $R$ be the component of $D \backslash\left\{\left(\bigcup_{i} r_{i}\right) \cup\left(\bigcup_{j} s_{j}\right)\right\}$ which contains the origin. For fixed small $\alpha$ and sufficiently small $\beta$, the size of the angle formed inside $R$ by 
the intersection of $s_{j}$ and $r_{j}$ is a monotone function of $\beta$ which varies from values above $\pi / 2$ to zero as $\beta$ tends to $\alpha$. Hence, for fixed small $\alpha$ there is a unique choice of $\beta$ such that the $4 \mathrm{~g}$ interior angles of the noneuclidean polygon $\bar{R}$ are each of size $\pi / 2 g$. For each $\alpha$ we will use this value of $\beta$. $\bar{R}=\bar{R}(\alpha)$ is a noneuclidean $4 g$-gon, the interior angles of which sum to $2 \pi$ and such that the $j$ th and $(j+2)$ nd sides are the same length for each $j$. Hence [4, volume II, p. 84] $\bar{R}(\alpha)$ is the fundamental region for $S(\alpha)$, a compact Riemann surface of genus $g$. We now show that for small $a$, size $(S(\alpha))>K$. Pick and fix a small positive number $\epsilon$. For $j=1,3, \cdots$, $2 g-1$, let $t_{j}$ be the noneuclidean straight line which connects $a_{j} \exp (i \epsilon)$ to $a_{j} \exp \left(-i_{\epsilon}\right)$. For small $\alpha$ the part of $\bar{R}(\alpha)$ separated from the origin by the $t_{j}$ corresponds to a collection of $g$ annuli $A_{1}, A_{3}, \cdots, A_{2 g-1}$ on $S(\alpha)$. For $j=1,3, \cdots, 2 g-1$, let $A_{j}^{\prime}$ be the annulus on $S(\alpha)$ obtained by removing from $A_{j}$ the intersection of $A_{j}$ with a small disk about the point of intersection of $s_{j}$ and $r_{j}$. The $A_{j}^{\prime}$ ar e disjoint and this set of annuli reduces $S(\alpha)$. To show $S(\alpha)>K$ it suffices to show $\left|A_{i}^{\prime}\right|_{S(\alpha)}>K$. However for fixed $\epsilon,\left|A_{i}\right|_{S(\alpha)}$ tends to infinity as a approaches 0 .

A similar construction gives surfaces of genus 1 of arbitrarily large size.

Corollary. Theorem 1.

Proof. Propositions 1 and 2 show that $R$ must be planar. The preliminary lemma of Heins' proof [2, Lemma 1] states that a Riemann surface which can be realized as an open planar subregion of a compact Riemann surface of positive genus is conformally equivalent to a bounded plane region.

4. Notes. A. Heins actually proved that surfaces which can be extended to certain highly symmetric surfaces must be planar. We have proved more than this. The surfaces of large size constructed in the proof of Proposition 2 are symmetric, however size $(S)$ is a continuous function of the conformal structure of $S$. More specifically, for a compact Riemann surface, the function size(.). is a continuous function on $T(S)$, the Teichmüller space of $S$. This follows from the realization of quasiconformal deformations of $S$ as quasiconformal deformations of the associated group of linear fractional maps of the disk [1, Chapter VI]. The surfaces which have nontrivial conformal automorphisms form a subset of $T(S)$ which has a dense complement [3]. These facts; together with Proposition 2, show that there are compact surfaces of arbitrarily large size which have no nontrivial conformal automorphisms.

B. The proof of Proposition 1 does not really use the univalence of the map $f$. All that is used is that if $\gamma_{1}$ and $\gamma_{2}$ are curves which represent a 
pair of homology classes with nonzero intersection number, then $f\left(\gamma_{1}\right)$ and $f\left(\gamma_{2}\right)$ have the same property. Hence Proposition 1, and thus also Theorem 1 , apply to a weaker notion of continuation of surfaces-one defined in terms of the action of the continuation map on the homology group. For example, Theorem 1 remains true if the "continuation" map is only required to be an analytic map which has the same action on the homotopy group as a (not necessarily analytic) unival ent map.

C. The idea in the proof of Theorem 1 is that there are surfaces on which a pair of homology classes with nonzero intersection number cannot consist entirely of curves which are short with respect to the Poincare metric. Analogous results can be proved using other geometric criteria which insure that such a pair of homology classes cannot consist entirely of curves which are short in some general sense. We outline briefly a specific version of such an approach. This version is derived from a recent proof of Heins' theorem by Professor J. Jenkins [5].

For an annulus $A$ in a Riemann surface $S$, let $L(A)$ be the extremal length of the family of locally rectifiable curves in $A$ which connect the two boundary components of $A$. Define size' $(S)=\sup \inf _{i} L\left(A_{i}\right) ;\left\{A_{i}\right\}$ reduces $S\}$. The analogs of Propositions 1 and 2 are both true for this new size function. The proof of the analog of Proposition 1 is similar to the proof of Proposition 1 although some technical complications arise. The analog of Proposition 2 is proved by constructing the appropriate surfaces. Let $T_{g}$ be the Riemann sphere with disjoint disks about the points $0,1, \cdots, g$ removed. Let $S_{g}$ be the double of $T_{g}$. $S_{g}$ is a compact Riemann surface of genus $g$ and size' $\left(S_{g}\right)$ can be made arbitrarily large by making the excised disks sufficiently small. Similar constructions give surfaces $S$ of infinite genus of various topological types with size'( $S)$ arbitrarily large size. Thus a stronger version of Proposition 2 is available for the function size' $(\cdot)$.

\section{BIBLIOGR AP HY}

1. L. Ahlfors, Lectures on quasiconformal mappings, Van Nostrand Math. Studies, no. 10, Van No strand, Princeton, N.J., 1966. MR 34 \#336.

2. M. Heins, A problem concerning the continuation of Riemann surfaces, Contributions to the Theory of Riemann Surfaces, Ann. of Math. Studies, no. 30, Princeton Univ. Press, Princeton, N.J., 1953, pp. 55-62. MR 15, 25.

3. H. Rauch, A transcendental view of the space of algebraic Riemann surfaces, Bull. Amer. Math. Soc. 71 (1965), 1-39. MR 35 \# 4403.

4. C. L. Siegel, Topics in complex function theory, Vols. I, II, Wiley, New York, 1971.

5. J. Jenkins, On a result of $M$. Heins (to appear). 63130

DEPARTMENT OF MATHEMATICS, WASHINGTON UNIVERSITY, ST. LOUIS, MISSOURI 